\title{
Pasos y movimientos retóricos del planteamiento del problema, del género protocolo de tesis de licenciatura, en estudiantes de Traducción de la Facultad de Idiomas, Universidad Autónoma de Baja California.
}

Steps and rhetorical moves in the problem statement of a thesis protocol carried out by B.A. Translation students from the Faculty of Languages-Ensenada at the Autonomous University of Baja California.

\author{
Eyder Gabriel Sima Lozano \\ Universidad Autónoma de Baja California (MÉXICO) \\ CE: eyder.sima@uabc.edu.mx ID ORCID: 0000-0002-1303-8919 \\ Jesús Eduardo Fong Flores \\ Universidad Autónoma de Baja California (MÉXICO) \\ Tatiana Estefanía Galván de la Fuente \\ Universidad Autónoma de Baja California (MÉXICO)
}

DOI: $\underline{10.32870 / \text { sincronia.axxiii.n76.36b19 }}$

(c) $\mathrm{BY} \cdot \mathrm{NC}$

Esta obra está bajo una Licencia Creative Commons Atribución-NoComercial 4.0 Internacional

Recibido: 25/02/2019

Revisado: 07/03/2019

Aprobado: 03/05/2019 


\section{RESUMEN}

Desde la Lingüística Sistémico-Funcional un movimiento retórico es definido como un segmento textual con un contenido proposicional y una intención comunicativa, dividido en pasos que tienen una meta específica (Swales, 1990, Nwogu, 1997). Siguiendo esta perspectiva teórica, el presente estudio tiene como objetivo identificar los movimientos retóricos y sus pasos en el planteamiento del problema del género, denominado protocolo de tesis, realizado por estudiantes de la Licenciatura en Traducción de la Facultad de Idiomas, UABC extensión Ensenada.

La metodología aplicada es de índole cualitativa de tipo inductiva, siguiendo el estilo descendente con auditoría del investigador, quien lleva a cabo diversas lecturas a fin de localizar los distintos movimientos y pasos retóricos.

Entre los resultados sobresalientes se encuentran que los estudiantes de traducción atienden a dos movimientos retóricos obligatorios: la fase histórica y la fase personal, en donde desarrollan la cuestión histórica del tema y realizan sus propuestas atendiendo al objetivo, las preguntas de investigación, entre otros pasos dentro del texto.

Palabras clave: Planteamiento del problema. Protocolo de tesis. Traducción. Pasos. Movimientos retóricos.

\section{Abstract:}

Seen from the perspective of systemic-functional linguistics, a rhetorical move is defined as a text segment with content proposal and a communicative intent, divided into steps with a specific objective (Swales, 1990, Nwogo, 1997). Following this theoretical perspective, the aim of the present study is to identify the rhetorical moves in the problem statement of a thesis protocol carried out by B.A. Translation students from the Faculty of Languages in Ensenada.

The methodology followed is qualitative in nature adhering to an inductive orientation; following a descendant style where the researcher carries out diverse reading in order to pin-point different rhetorical moves. 
The most salient data findings are that Translation students focus on two obligated rhetorical move- the historical and personal phases, where they develop both the historical and the proposal itself, tending to the objectives, research questions, as well as other steps within the text.

Keywords: Problem statement. Thesis protocol. Translation. Steps. Rhetorical moves.

\section{Introducción.}

Desde el Análisis del Discurso, la Lingüística Sistémico-Funcional y la Semiótica Social en sus relaciones con la Lingüística Aplicada, diversos estudios se han realizado para explicar la redacción de géneros académicos a fin de encontrar modelos de producción pedagógica para los campos disciplinares en cuestión. Autores como: (Castro, 2010; Hernández, 2011; Moyano, 2007) caminan en esa dirección. Además, se analizan los textos para identificar estructuras de los productos según su género y que éstas sirvan como guías de redacción especializada en la escritura de ensayos, artículos, capítulos de libros, tesis y partes fundamentales de estos ejemplos (Perales, Sima y Valdez, 2014; Castro, Hernández y Sánchez, 2010).

Entre las perspectivas de análisis, los movimientos retóricos tienen relevancia al ser fuente de explicación de los procedimientos y estructuras que le dan forma a los géneros, particularmente académicos. Desde la Lingüística Sistémico-Funcional un movimiento retórico es un segmento textual, con un contenido proposicional y una intención, atendiendo a diferentes propósitos de la construcción textual (Swales, 1990, Nwogu, 1997). En tanto, el paso retórico constituye una parte del movimiento retórico que atiende a funciones más específicas. Asimismo, Perales, Sima y Valdez (2014) explicaron la utilidad del estudio de movimientos y pasos retóricos para la caracterización de las regulaciones y variaciones discursivas de textos pertenecientes a un género determinado.

La producción del género académico de tesis, previo a su desarrollo, plantea la redacción de un proyecto específico y de interés del estudiante en formación. En la Licenciatura de Traducción, adscrita a la Facultad de Idiomas de la Universidad Autónoma de Baja California, México, se oferta la asignatura Seminario de Investigación. La competencia general a desarrollar se centra en el 
desarrollo de un protocolo de tesis que deberá entregar a partir de un punto de vista crítico y argumentativo donde fundamente la problemática a investigar. El planteamiento del problema es la parte fundamental que muestra los movimientos y estructuras principales para el desarrollo del protocolo, en éste se encuentra la problematización que permite en este nivel comprender hacia dónde se dirige la propuesta.

Por lo anterior, planteamos la siguiente pregunta que guiará el análisis: ¿cuáles son los movimientos retóricos para la redacción del planteamiento del problema en protocolos de tesis del campo de la traducción de la Facultad de Idiomas, UABC? En consonancia con la pregunta descrita anteriormente, el objetivo es identificar los movimientos retóricos en el planteamiento del problema, del género protocolo de tesis, realizado por estudiantes de la Licenciatura en Traducción de la Facultad de Idiomas, UABC.

Es importante puntualizar que se decide trabajar con el programa educativo Licenciatura en Traducción a condición de la amplia matricula y, al reflexionar la importancia que tiene al ser un programa reconocido y acreditado a nivel nacional que busca cubrir ciertos estándares educativos. Se espera, además, que el profesional de la traducción esté familiarizado con la redacción de géneros académicos y sea capaz de producir diversos textos, puesto que una de sus tareas laborales es la traducción de una lengua hacia otra en géneros variados: científico, literario, comercial, legal, religioso, publicitario, etc.

\section{Contexto}

La producción del género académico de la tesis, previo a su desarrollo, plantea la redacción de un proyecto de tesis, conocido también como protocolo de tesis o anteproyecto según las normativas en diversas universidades en el nivel licenciatura como en posgrado. A nivel licenciatura, contexto de estudio para la presente investigación, existen variadas modalidades de titulación que permiten la obtención de grado, tales como: programa educativo de calidad, promedio general, informe de prácticas profesionales, entre otras. Las cuales han generado un abandono ante el interés de la redacción de la tesis, la cual fue durante años la única forma para alcanzar la titulación y la 
eficiencia terminal de los programas educativos. Importante reconocer que han sido escasas las ocasiones que se ha optado por esta modalidad, la cual representa un cambio de percepciones ante la investigación y la tesis misma.

Actualmente, los programas de licenciatura, sobre todo en el campo de las humanidades, presentan variantes sobre la impartición de una asignatura dirigida a la redacción del protocolo de tesis, misma que recibe nombres diferenciados: seminario de titulación, seminario de investigación, seminario de tesis, redacción de proyecto de tesis. En tales cursos, la búsqueda se orienta hacia la comprensión de modalidades y formas de titulación, la redacción de un protocolo de tesis con miras al desarrollo de la tesis como una opción de investigación, estructura y redacción de la tesis, siendo que en algunos casos estos cursos son seriados, y en otros se imparten durante un semestre únicamente.

En la Facultad de Idiomas de la Universidad Autónoma de Baja California, se ofertan actualmente dos programas educativos a nivel licenciatura: La licenciatura en Enseñanza de Lenguas y la Licenciatura en Traducción. Ambos programas educativos cuentan con asignaturas vinculadas con la investigación, el primero, a través de asignaturas como Investigación Educativa, Intervención en la Enseñanza de Lenguas, entre otras que, de una forma u otra atienden a la Investigación. Por otro lado, el Programa Educativo Licenciatura en Traducción contempla la formación en la investigación a partir de una formación transversal donde se puntualiza, en este caso, en la asignatura Seminario de Investigación, correspondiente a la etapa terminal del plan de estudios 2006-2.

En el séptimo semestre de la licenciatura en Traducción, los estudiantes cursan obligatoriamente la asignatura de Seminario de Investigación; presentan como producto terminal un protocolo formal de tesis, el cual consta de diversos apartados tales como: título, planteamiento del problema, justificación, antecedentes, marco teórico, metodología, tipo de investigación, paradigma o enfoque, alcance de la investigación, cronograma, índice capitular, bibliografía, corpus.

Al contemplar la perspectiva e interpretación de los docentes ante la competencia general del curso, se espera que el alcance atienda al desarrollo de un proyecto de tesis, que describa la 
intención del autor y sustente su relevancia desde la perspectiva crítica-analítica. Sin embargo, la carta descriptiva de forma caótica enuncia que el objetivo principal son las partes que componen a la tesis, por lo que no existe claridad entre lo que se espera como resultado final. En la práctica nos encontramos, únicamente como producto, un protocolo de tesis.

\section{Marco teórico}

Siguiendo la afirmación de Halliday (1975) en el sentido de que el lenguaje es un camino para la formación de los saberes al ser un proceso en el que las experiencias se convierten en conocimiento, se justifica el análisis del lenguaje de los expertos y participantes de un campo disciplinar, a fin de identificar los estilos y el proceso retórico que definen la manera en que deben ser presentados los géneros que son sensibles a una planificación textual, particular, como lo son los de tipo académico. En otras palabras, el texto es objeto de estudio tal como la afirma Van Dijk (1978).

En la teoría de la Lingüística Sistémico-Funcional encontramos un cobijo para la explicación de lo que entendemos por género, concepto que requerimos asentar en este estudio, ya que nos advocamos al análisis del protocolo de tesis, el cual vendría a ser un género con sus variables, estructuras, tipologías y una forma de ser construido por sus autores. En la Lingüística del texto, la cual forma parte de la Lingüística Sistémico-Funcional, la propuesta de género ha atendido más a la definición de tipos y formas en las que se producen los textos, atendiendo predominantemente a la estructura gramatical (Ciapuscio, 2005) pero se ha dejado a un lado a los participantes que también son capaces de adherir estructuras semióticas con carácter de significado social, lo que resulta adecuado en la Lingüística Sistémico-Funcional (LSF) que toma en cuenta el punto de vista de los que se adhieren al género.

Así, siguiendo la propuesta de la Escuela de Sidney en su relación con la Lingüística Sistémico-Funcional, el género lo definimos como una actividad de naturaleza social con una estructura organizada que sigue objetivos, según Martin (1992). Esto nos permite tomar en cuenta 
los criterios para la formación y definición del protocolo de tesis, según los participantes del corpus del presente trabajo.

El siguiente concepto importante para definir es el de movimiento retórico. Líneas arriba en la introducción mencionamos que se conceptualiza como un segmento textual que atiende a diferentes propósitos (Nwogu, 1997). Siendo el elemento teórico más relevante para explicar la construcción de una estructura del protocolo de tesis. Cabe agregar que su uso se orienta a la indagación de las estructuras que componen al género a fin de observar las prácticas textuales de un campo disciplinar y delimitar las funciones que ciertas estructuras cumplen en el género analizado. En esta dirección, nos apropiamos del término denominado etapas funcionales de acuerdo a Eggins y Martin (2000) para expresar una forma sinónima que define a los movimientos retóricos, ya que de acuerdo a esta propuesta, cada segmento textual viene a constituir una etapa en la que median propósitos y metas comunicativas de tipo global.

Pero los movimientos retóricos pueden a su vez fragmentarse en elementos menores, conformando segmentos textuales de menor extensión, conocidos como pasos. Así, reconocemos que contemplan una categoría menor, dado que su carácter es local y forman parte de la comunicación global de los movimientos retóricos, actúan a nivel micro, colaborando para el propósito comunicativo del movimiento retórico.

Por otra parte, un movimiento retórico tiene la posibilidad de ser opcional y obligatorio, afectando también a los pasos. Tanto movimientos y pasos están condicionados a las expectativas del género producido. Cabe mencionar que la frecuencia es un criterio que permite establecer como norma la producción de carácter obligatoria.

Pasamos ahora a definir el género del protocolo de tesis. Consideraremos la literatura que proponen autores como Muñoz, quien habla de propuesta de tesis (1998, p.109): "el punto de partida para iniciar una investigación y desarrollar con ella un tema que culmine en una tesis es la presentación formal, por escrito, de la propuesta de tesis". Explica que debiera ser clara, sencilla a fin de evaluar la viabilidad del trabajo para que a juicio del asesor se analice la posible realización de la tesis. 
En tanto, Hernández, Fernández y Baptista (2014) no plantean la redacción de una propuesta previa a la investigación de un tema, dado que se concentran en los pasos que debiera llevar el proceso investigativo de forma general. Así lo expresa: "en este capítulo se plantea la forma en que se inician las investigaciones de cualquier tipo mediante ideas" (2014, p.22). Pero hacen una ligera mención sobre el protocolo cuando abordan el planteamiento del problema.

Melpopharma (2017), una organización que se asume como gestora del conocimiento entre la docencia y la investigación, define el protocolo de tesis como la fase previa al diseño de una investigación, considerando que su extensión no exceda las 30 hojas y contenga elementos como título, pregunta de investigación, antecedentes, justificación de la investigación, objetivos generales y específicos, metodología, cronograma y anexos.

Al referir brevemente qué se entiende por protocolo de tesis, pasamos ahora a explicar el elemento que nos interesa para su análisis que es el planteamiento del problema. Encontramos que Hernández, Fernández y Baptista $(2014$, p.48) lo ubican como un elemento del protocolo de tesis: "el planteamiento del problema siempre se incluye en las propuestas o protocolos y los reportes o informes de resultados".

Los autores exponen que el planteamiento del problema es el centro, el corazón de la investigación, pues es el elemento que dicta o define los métodos para la investigación. Asimismo, su fin es afinar y darle estructura formal a la investigación poniendo en desarrollo cinco elementos: objetivos, preguntas, justificación, viabilidad y evaluación de las deficiencias.

En tanto, Muñoz (1998) expone que en el planteamiento del tema de la investigación existe un punto que se llama proposición de tema, que sería lo más similar al planteamiento del problema. En este se busca que el alumno se centre en una propuesta concreta que ubique el planteamiento global de la investigación: "y lo más claramente posible el aspecto sobresaliente que buscará satisfacer con su trabajo, es decir, la base de su propuesta de investigación" (Muñoz, 1998, p.71).

Por su parte, el Centro de Recursos para la Escritura Académica (2019) define que el planteamiento del problema es el inicio de una investigación donde surgen las preguntas: ¿Qué tema se quiere tomar como eje de la investigación? ¿De dónde se parte y a dónde se pretende 
llegar? Con la primera pregunta se espera un conocimiento previo por el tesista y con la segunda, la construcción del contexto y hacer uso de las herramientas al alcance para llegar a un fin. Se espera que contenga los siguientes elementos: contexto, definición del problema, preguntas de investigación, objetivo general, justificación, beneficios esperados.

Por su parte, El manual de la Universidad Veracruzana (S/F) habla del planteamiento de la investigación y lo define como el primer elemento hacia la elaboración del protocolo:

En este apartado, se hace referencia a la formulación del problema a investigar. Es el primer paso en la elaboración de un protocolo, ya que de esto se derivan las acciones necesarias para determinar lo que se va a investigar. Debe tenerse en cuenta que la selección del problema requerirá un respaldo teórico y práctico. Es necesario que la definición del problema de investigación sea clara y precisa puesto que de ella dependerá la formulación adecuada de abordarlo. (Universidad Veracruzana, S/F, p.3).

Así, considerando las definiciones y propuestas de algunos autores con respecto al planteamiento del problema, observamos que existen marcadas diferencias para explicar en qué consiste el elemento en cuestión. Por nuestra parte, consideraremos y estaremos de acuerdo en que el planteamiento del problema es la sección central que expone los argumentos principales que delinean el horizonte de la investigación, proponiendo lo que se investigará. Los objetivos y las preguntas de investigación forman parte del elemento. Los objetivos manifiestan el fin del trabajo de forma sintética y son una delimitación, en tanto las preguntas son el punto de partida del análisis y el planteamiento de lo que se busca responder.

\section{La metodología de análisis}

Este estudio sigue una metodología cualitativa, de tipo inductiva, porque no se incorporan movimientos retóricos a partir de otros estudios, sino que se analizan los textos siguiendo las propuestas de los autores quienes desarrollan los protocolos de tesis en cuestión. 
La muestra está conformada por un conjunto de veintisiete protocolos de tesis, los cuales corresponden a tres generaciones, tres grupos de la Licenciatura en Traducción de la Facultad de Idiomas, Ensenada. La forma en la que se obtuvieron fue a través de una solicitud de permiso a los estudiantes que fueron contactados e informados de los objetivos del presente estudio. En una carta autorizada, ellos asentaron que estaban de acuerdo que su trabajo fuera parte del análisis y se guardaría la confidencialidad de sus identidades. Los autores de la presente investigación seguimos la propuesta de Ackland (2009), quien propone el estilo descendente con auditoría del investigador. Este método propone que se realicen diversas lecturas del texto para encontrar los movimientos, segmentándolo en partes a partir de diversas oraciones que juntas tienen un contenido proposicional. Biber, Connor y Upton (2007) señalan que el análisis se realiza en diferentes periodos, en donde los investigadores intervienen codificando los movimientos retóricos, realizando además, comparaciones constantes.

Por lo anterior, este método nos parece más adecuado que si optáramos por encontrar movimientos retóricos similares de otros estudios, ya que la naturaleza de nuestro material proviene de fuentes no publicables y que son usadas para una forma de evaluar una asignatura. Así, una vez recolectado todo el material para el objeto de investigación, nos dimos a la tarea de llevar a cabo las lecturas, comparaciones y crear una etiqueta para nombrar al movimiento retórico en cuestión.

\section{Movimientos retóricos en el planteamiento del problema de protocolos de tesis}

Para responder a la pregunta de investigación planteada en la introducción: ¿cuáles son los movimientos retóricos para la redacción del planteamiento del problema en protocolos de tesis del campo de la traducción de la Facultad de Idiomas, UABC?

En la revisión del corpus encontramos que existen dos secciones básicas que siempre están presentes: la primera se nombra como fase histórica y la segunda denominada como fase personal. En la fase histórica es común encontrar la contextualización y problematización del tema, pues en éste se expone la situación histórica. En tanto, en la segunda fase se localiza la propuesta en la que 
el autor enuncia los propósitos que rigen su investigación, además, al final de la sección es característico la presentación de los objetivos y las preguntas de investigación. Lo anterior se corresponde con los apuntes del docente que imparte la asignatura y la estructura esperada para la presentación de esta sección en el protocolo de tesis.

Considerando que en nuestra definición conceptual se define el movimiento retórico como un segmento textual que atiende a propósitos específicos, encontramos que el planteamiento del problema podría considerarse un tipo de macromovida o macromovimiento de acuerdo con Parodi (2010), ya que este presenta una intencionalidad comunicativa más amplia.

Luego, el planteamiento del problema se forma de dos movimientos retóricos obligatorios: la fase histórica y la fase personal, apostamos por esta explicación, debido a que cada una de ellas aportan sus propios pasos articulados a un propósito más específico y hacia objetivos diferenciados: la histórica atiende al pasado y presente del tema, su situación actual, contextualización, breves referencias a autores, usando tiempo gramatical en pasado y presente; en tanto, la personal se dirige a que el estudiante proponga lo que hará con el tema. Así, la fase histórica y la fase personal constituyen movimientos retóricos, segmentaciones discursivas que comunican un propósito. Por lo tanto, nuestros dos movimientos retóricos representativos del problema se constituyen en pasos específicos. De acuerdo a la propuesta teórica un paso es una realización diferenciada que aporta una intención comunicativa específica a la estructura del movimiento retórico.

Comenzando con los pasos del movimiento retórico fase histórica, encontramos los siguientes a nivel frecuencia, presentamos posteriormente ejemplos de ellos y breves notas:

\begin{tabular}{|l|c|}
\hline \multicolumn{1}{|c|}{ Movimiento } & Frecuencia \\
\hline Definición del tema & $27 / 27$ \\
\hline Posicionamiento temporal del tema & $13 / 27$ \\
\hline $\begin{array}{l}\text { Descripción en base a una referencia de la } \\
\text { literatura }\end{array}$ & $2 / 27$ \\
\hline
\end{tabular}




\begin{tabular}{|l|c|}
\hline $\begin{array}{l}\text { Descripción en base a una cita textual } \\
\text { histórica }\end{array}$ & $1 / 27$ \\
\hline $\begin{array}{l}\text { Explicación del tema a partir de la revisión } \\
\text { de la literatura }\end{array}$ & $10 / 27$ \\
\hline $\begin{array}{l}\text { Fundamentación a partir de una cita } \\
\text { textual }\end{array}$ & $3 / 27$ \\
\hline Descripción de un escenario & $9 / 24$ \\
\hline Descripción de un contexto & $16 / 24$ \\
\hline Deber del tema hacia una realización & $23 / 27$ \\
\hline Problematización del tema en diversas & $15 / 27$ \\
\hline áreas & \\
\hline Cierre de la sección con expectativas del \\
tema
\end{tabular}

A) Definición del tema: el autor explica brevemente en qué consiste la temática central del trabajo, como una forma de definir brevemente el problema para dejar claro de qué trata este aspecto que posteriormente se analizará. Se tiende a centrar esta explicación en un concepto o término principal que guiará la investigación. Es de tipo obligatorio, pues aparece totalmente en cada uno de los trabajos con 27 frecuencias. Por lo general, ocupa la primera posición y las primeras líneas de la sección, como en el primer ejemplo, pero puede presentarse tardíamente como aparece en el segundo ejemplo, por lo que es un movimiento obligatorio pero no con presencia obligatoria en los primeros párrafos del movimiento retórico:

Los diccionarios son una herramienta esencial, primordial e imprescindible para los traductores. Con el paso del tiempo, se han ido mejorando y adaptando de acuerdo a la cultura y nuevos vocabularios del idioma en el que se presentan. Surgieron poco después de 
la invención de la escritura. Iniciaron siendo solamente monolingües y eventualmente nacieron los diccionarios enciclopédicos y los especializados. (1).

Una de las características que distinguen a esta traducción del resto es su libertad de interpretación de los textos a traducir y esto permite un sinnúmero de conceptos que pueden presentarse en diversas variedades. Tal es el caso del adjetivo; en el contexto bilingüe de los idiomas inglés y español el adjetivo es el elemento gramatical que más libertad de traducciones goza, característica que evita que dos traductores lleguen a una misma traducción literaria de un único texto. (2).

B) Posicionamiento temporal del tema: el autor toma un punto de partida histórico para situar el tema en alguna fecha exacta o época para explicar el origen del tema, la problematización o darle la relevancia al fenómeno en eventos temporales que desea resaltar. Su posicionamiento es dentro de los primeros párrafos de la sección, a fin de centrar el tema dentro de la historia. No se constituye en un paso obligatorio, tiende a ser de tipo opcional, pues del corpus analizado solo 13 de 27 lo presenta.

Uno de los primeros diccionarios especializados en medicina registrados es del año 1812 en el idioma francés. Poco a poco empezaron a nacer bases de datos terminológicamente especializados creados por uno o varios terminólogos con ayuda de traductores. Ahora, esas bases de datos (también conocidas como "corpus") ya pueden ser encontradas en la web. (1)

A principios de 1950, la investigación sobre la traducción automática inició teniendo altas expectativas en sus resultados, es decir, se creía que la calidad de las traducciones podía ser igual a las traducciones realizadas por humanos. Fue hasta 1966 cuando esa esperanza desapareció y, en su lugar, hubo un enfoque en la CAT (Computer-Assisted Translation). (18). 
C) Descripción en base a una referencia de la literatura: se describe el tema haciendo referencia a autores, pero no sobre la base teórica, sino sobre algún evento histórico o época relacionada con el tema, con apenas 2 frecuencias encontradas es un paso opcional:

La segunda opción fue recurrir al subtitulaje, pero se descartó de inmediato debido al gran número de analfabetas que había en aquella época. (Casillas, 2009) (13).

D) Descripción en base a una cita textual histórica: se plantea el segmento considerando su relación con un evento histórico, no necesariamente con una orientación teórica, la cita se orienta hacia la ubicación del tema en el tiempo para proporcionar fuerza al segmento. Podría ser considerado una derivación del anterior movimiento retórico posicionamiento temporal del tema. Apareció únicamente con una frecuencia, por lo que es de tipo opcional:

Algunos ya pensaban que el uso de los intertítulos hacía que las películas fueran lentas y distractoras:

De todas formas, los intertítulos fueron cruciales en la exportación del cine. En lugar de simplemente traducirse, se adaptaban creativamente para las distintas audiencias, y se modificaban nombres, lugares y otros detalles culturales para hacerlos más accesibles. Al final de la era muda, se rehacían intertítulos en Estados Unidos para 36 mercados lingüísticos distintos. Sin embargo, cuando llegaron las películas sonoras, Hollywood siguió el ejemplo de sus rivales europeos produciendo versiones en lengua extranjera de sus películas más prestigiosas. (BASSOFIA, 2013). (5).

E) Explicación del tema a partir de la revisión de la Literatura: el autor proporciona una referencia para situar el tema dentro de los estudios con los que presenta una relación. Siendo un paso de tipo opcional, su frecuencia no es muy alta, se presentaron 10 frecuencias con la particularidad de que 7 son propios de 7 protocolos, es decir, una vez por cada proyecto. Pero en un trabajo se presentaron 3 tipos de este paso. 
Según Ramos (2001) La traducción se convirtió en el instrumento de actualización de las tradiciones remotas e inmediatas y en canal de diálogo entre los movimientos artísticos nacionales. A partir de la segunda década del siglo se intenta renovar el canon nacional e internacional, momento en el que las traducciones desempeñan un importante papel en la formación crítica y estética de las élites culturales. (14).

F) Fundamentación a partir de una cita textual: siguiendo de forma similar con el anterior paso, ahora el autor fundamenta el tema presentando una cita textual con la que existe una relación temática. Se presentaron únicamente 3 casos, por lo que inferimos que existe menos preferencia por la cita que por la paráfrasis.

Por otro lado, el aspecto psicolingüístico consiste en la probabilidad en la que el hablante pueda desenvolverse de manera comunicativa en las dos lenguas.

El bilingüismo consiste en la facultad que posee un individuo de saber expresarse en una segunda lengua adaptándose fielmente a los conceptos y estructuras propias de la misma sin parafrasear la lengua nativa. La persona bilingüe posee la facultad de saber expresarse en cualquiera de las dos lenguas sin dificultad cada vez que surge la ocasión (Titone, 1976: 13).

G) Descripción de un escenario: se describe el escenario de trabajo, planteando acontecimientos históricos, eventos, fechas, referencias que le han dado formación e identidad al lugar o espacio en donde ocurre el tema. Aparece como un paso de tipo optativo con 9 frecuencias. Se encuentra ligado al siguiente paso, descripción de un contexto:

En el contexto local de la ciudad de Ensenada, la primera asociación de traductores y las reuniones semanales llevadas a cabo por personal de la facultad de idiomas, nos permite observar la repartición de trabajo entre los miembros, así como las especialidades que experimentan mayor demanda según los idiomas envueltos en el proceso. (4). 
CICESE (Centro de Investigación Científica y de Educación Superior de Ensenada Baja California) es un centro público de investigación que ha trabajado para descentralizar las actividades científicas del país por alrededor de 40 años. Uno de sus principales objetivos es el generar conocimiento científico. Por la naturaleza científica de esta institución y por su objetivo de divulgación científica, constantemente se redactan artículos acerca de las diversas temáticas y problemáticas que se presentan en el país en relación con las ciencias. Sus investigaciones proponen soluciones a problemas de gran importancia para el país. CICESE es una institución de renombre y de gran impacto para la comunidad mexicana. Se busca siempre trabajo y soluciones de calidad. (6).

H) Descripción de un contexto: A diferencia del paso anterior, pero de forma similar se describe un escenario de carácter abstracto, sobre todo cuando se trata de contextualizar textos literarios o plataformas como Netflix que serán relevantes para el desarrollo del tema. Apareció en el corpus con 16 frecuencias, lo que indica que la mayoría de los temas se enfocan en datos que surgirán de la investigación documental:

Dentro del contexto de la novela de ciencia ficción "The Phoenix Conspiracy", originalmente escrita en el idioma inglés, existen diversos adjetivos que de no ser traducidos adecuadamente pueden provocar una lectura torpe y de poca calidad, por lo que es necesario delimitar cuáles son las restricciones y libertades del traductor literario respecto al uso del adjetivo. (2).

I)Deber del tema hacia una realización: este movimiento pretende ser una enunciación sobre el deber hacia algo por parte del tema en cuestión. Puede usar el verbo deber o no, pero siempre su intención es asegurar una obligación de la problemática analizada. Es un paso prácticamente obligatorio, debido a que se presentó con una frecuencia de 23 veces en el corpus.

Con el acceso a internet y el excesivo contenido de series y películas existente, el trabajo para los traductores que trabajan subtitulando es demasiado, pero no siempre es el que el 
usuario desea tener; en ocasiones, el usuario desea ver alguna serie de algunos años atrás que no le interesa a la población en general o a una cadena de televisión, lo cual lleva a los aficionados que conocen el idioma original de la serie o película a crear sus propios subtítulos. (8).

Los diccionarios al igual que la lengua, evolucionan. Dicho esto, un traductor también debe desarrollarse y educarse acerca de nuevos avances tecnológicos relacionados a su profesión para lograr que el producto de sus traducciones sea absolutamente fiel a todos los aspectos de la lengua meta. (1).

J) Problematización del tema en diversas áreas: en este ámbito el autor vincula el tema que tratará desde diversos ángulos, hace una relación de las diversas temáticas o problemáticas con las que se vincula el tópico principal, con una frecuencia de 15 representaciones en los protocolos puede considerarse un paso de tipo semiobligatorio en la estructura del protocolo.

Queda claro que el humor tiene sus variantes y que dentro del universal hay otros tipos de humor. Y cada cultura tiene sus chistes y su forma de crearlos, de contarlos, e incluso, de transmitirlos a otras culturas. (20).

Cada año producen más cantidades de la película, series, animaciones y otros tipos de videos que sus motivos son para entretenimiento, y eso lleva a cabo la necesidad de traductores técnicos profesionales para subtitular en diversos idiomas. Existen varias dificultades al realizarlos ya que sea por cuestión técnica o alguna otra razón, pero uno de las dificultades más batallado es la cuestión cultural de idioma, la adaptación. (15).

K) Cierre de la sección con expectativas del tema: en este caso, el autor realiza un cierre anticipando, previendo lo que se podría lograr con el tema hacia el futuro. Es un paso de tipo opcional, su frecuencia se redujo solamente a 8 representaciones. 
Dentro del contexto de la novela de ciencia ficción "The Phoenix Conspiracy", originalmente escrita en el idioma inglés, existen diversos adjetivos que de no ser traducidos adecuadamente pueden provocar una lectura torpe y de poca calidad, por lo que es necesario delimitar cuáles son las restricciones y libertades del traductor literario respecto al uso del adjetivo.(2).

De esta manera, el tema incide la necesidad de estudiar cómo adaptar el lado cultural al momento de traducir subtítulos para que tenga mismo efecto en la lengua llegada. (15).

Pasamos ahora a explicar la construcción del movimiento fase personal y sus respectivos pasos, encontrándose los siguientes:

\begin{tabular}{|l|c|}
\hline \multicolumn{1}{|c|}{ Movimiento } & Frecuencia \\
\hline Propuesta enunciativa & $22 / 27$ \\
\hline Enunciación afirmativa & $3 / 27$ \\
\hline $\begin{array}{l}\text { Disciplina o noción conceptual que } \\
\text { fundamenta el trabajo }\end{array}$ & $11 / 27$ \\
\hline Referencia a otros trabajos & $2 / 27$ \\
\hline Disciplina o método desde el que se & $3 / 27$ \\
obtendrán los datos & $5 / 27$ \\
\hline Descripción de un escenario & $27 / 27$ \\
\hline Enunciación del objetivo general & $21 / 27$ \\
\hline Enunciación de objetivos particulares & $26 / 27$ \\
\hline Enunciación de las preguntas de & \\
\hline investigación & \\
\hline
\end{tabular}


A) Propuesta enunciativa: este es un movimiento muy amplio, con 22 frecuencias aparece constantemente referido en esta segunda parte del planteamiento del problema, predominantemente aparece desde el inicio de la fase personal, pero no siempre es así, ya que puede aparecer en medio y en las partes finales de la sección. Puede considerarse casi de tipo obligatorio, al tener 22 frecuencias. Se redacta como un tipo de objetivo, sin serlo formalmente, usa el tiempo gramatical en futuro. En algunos textos, cuando el autor no redacta la forma en futuro, se presenta en infinitivo: "es importante conocer". En ocasiones su extensión es breve, solo a partir de un párrafo, pero en otros casos implica varias propuestas a partir de diversas acciones desarrolladas hasta en tres párrafos, expresadas en los verbos como: "se propone", "se realizará", "se llevará a cabo", "haremos", "investigaré", "realizaré", por lo que puede constituir un macropaso:

Con esta investigación, se espera identificar los factores positivos y negativos para el traductor y la empresa/compañía, que surgen cuando es utilizada la traducción comercial como "impulso" para la empresa. Debido a que nos encontramos en un mundo totalmente globalizado, donde se vuelve necesario crecer económicamente para mejorar la calidad de vida. (26).

Esta investigación se enfoca en el campo de la traducción multimedia, se pretende mostrar los procesos traductológicos del doblaje y subtitulaje dado que los procesos son diferentes en cada uno de ellos, así como también sus restricciones. (12).

Cuando no aparece, es porque permite la representación del siguiente paso, ya que el autor expresa su intención de realizar una propuesta, pero a través de una afirmación que se presenta más como tipo descriptiva, muy parecida a la definición del tema, paso de la anterior sección, Fase histórica.

B) Enunciación afirmativa: este paso es una derivación del anterior, se presenta cuando el autor no establece una propuesta a futuro para desarrollar su investigación. De tipo 
opcional únicamente se presenta en 3 ocasiones. Se limita a la descripción del estado del tema, como si ésta llegara a ser su propuesta, aunque no es frecuente aparece en varios trabajos del corpus, ya sea como forma única o combinada con el paso anterior:

Muchas veces estos factores responden más a necesidades y aspectos de la producción que a la preferencia de la audiencia. Las técnicas de traducción audiovisual han avanzado junto con la industria audiovisual y los alumnos deben estar familiarizados con esos cambios si es que quieren dedicarse a la traducción audiovisual. [...]Para lograr la propuesta se verán los programas de diferentes escuelas en donde también se enseñe la traducción multimedia y ver qué se puede rescatar de cada programa. (11).

C) Disciplina o noción conceptual que fundamenta el trabajo: el autor enuncia el área o áreas disciplinares que le dan fundamento al estudio, el punto de partida disciplinar en el que ubica a su investigación, apareció con 11 frecuencias, siendo un paso de tipo opcional. En ocasiones se fundamenta a partir de la presentación de un constructo teórico como sucede en el tercer ejemplo.

A partir de la revisión de los procesos de adquisición en los niños de 10 a 15 años, se propone realizar una investigación centrada en la forma en la que estos niños adquieren estos préstamos y si la adquisición de estos repercute en el conocimiento de su idioma o del español. (21).

En el siguiente trabajo que se encuentra dentro del campo de "sociolingüística", se hará una comparación del lenguaje de la película "Alicia en el país de las maravillas" dependiendo de sus respectivas épocas para analizar la forma en la que el habla formal ha ido evolucionando debido a factores que influyeron en el medio. (22).

La traducción es el traspaso de manera oral o escrita de información de una lengua origen a una lengua meta. La localización es la adaptación de información a una lengua meta por regiones, por ejemplo: el subtitulaje de una película cuya lengua origen sea el inglés y lengua 
meta sea el español; primeramente, habrá que saber a qué parte se pretende llegar, si es que se pretendiera llegar al español hablado en Latinoamérica o al castellano. (5).

D) Referencia a otros trabajos: este paso presenta referencias de autores con los que se vincula el trabajo. Limitado únicamente a dos frecuencias es un paso de tipo opcional:

Como lo menciona Lachat (2011) de la Universidad de Granada; enseñar a traducir textos audiovisuales es enseñar a aplicar estrategias de traducción para resolver problemas de traducción audiovisuales. (11).

Como dice Zabalbeascoa en uno de sus tantos trabajos de 1994, 1996 ó 2005, por citar sólo algunos. (20).

E) Disciplina o método desde el que se obtendrán los datos: en este paso opcional con 3 frecuencias que formaría una subdivisión del anterior, el autor enuncia un campo disciplinar o una fuente desde el que obtendrá los datos de su trabajo, pero no es el campo disciplinar que guiará teóricamente su investigación, simplemente es un medio para la obtención de la muestra de análisis:

El campo disciplinar en el que vamos a indagar es el campo de la medicina. Creemos que, al ser ésta un área bastante compleja de traducir, es con mayor razón el área que más fuentes de terminología debería tener. (1).

Se pretende revisar la carta descriptiva existente de la materia Traducción Multimedia para rescatar las partes más importantes y así armar una nueva optativa como segunda parte para reforzar y adquirir nuevos conocimientos. (11).

F) Descripción de un escenario: el autor hace referencia al escenario en dónde obtendrá los datos de su propuesta. Es el espacio, medio, lugar en donde obtendrá los datos que posteriormente serán objeto de análisis. Constituye un paso opcional con 5 frecuencias: 
Ensenada es un punto de encuentro de distintas culturas. Es un puerto y centro turístico al que llegan constantemente personas de procedencia extranjera, de los cuales arriban en cruceros cada semana. (17)

Como se mencionó líneas arriba, el trabajo estudia el contexto de la comunidad oaxaqueña, en particular en los niños que por su edad pueden tener una mayor influencia hacia los préstamos, dichos niños habitan en la cuidad de ensenada. Debido a su influencia con los estadounidenses por trabajar vendiendo productos en las calles, lograr determinar e identificar si influye en ellos o si adquieren prestamos de estas personas. (21).

G) Enunciación del objetivo general: este es por lo general el antepenúltimo o paso de tipo obligatorio de la fase personal del planteamiento del problema, aparece en todos los trabajos analizados con 27 frecuencias. Suele aparecer un solo objetivo redactado en infinitivo. En algunos casos son parte de la narración de la propuesta, es decir, aparecen incrustados en el texto, pero es escaso este estilo:

El primer análisis pretende analizar la fluidez de los elementos dentro del contexto general, mientras que el segundo análisis pretende determinar si existen variables que puedan reemplazar la traducción original y cómo dicho reemplazo, si existe, cambiará el significado de la idea original. Por último, y en consideración con los objetivos ya mencionados, se reflexionarán los resultados para llegar a una resolución para responder las incógnitas que a continuación se plantean. (2).

El objetivo de esta tesis es identificar las problemáticas de los intérpretes mixtecos en el ámbito educacional en la comunidad de Maneadero, Ensenada. (3).

Por tales motivos, el objetivo de este trabajo es proponer la creación de un paisaje lingüístico que incorpore el español, inglés y lenguas indígenas en letreros de áreas turísticas del 
municipio de Ensenada, ya que esta área geográfica cuenta con hablantes de estas lenguas y sus variantes. (17).

Por lo general, los objetivos aparecen separados de la propuesta con un aviso previo de que a continuación o seguidamente aparecerán los objetivos. La función principal del objetivo es la enunciación de los fines y metas de la investigación, hacia donde se dirigen y quieren llegar:

Objetivo general: Identificar el proceso de adquisición de préstamos del inglés en niños migrantes oaxaqueños (21).

H) Enunciación de objetivos particulares: Al igual que el objetivo general aparecen redactados en infinitivo. Suelen aparecer dos como máximo. Sus fines son apoyar específicamente las metas particulares de lo que se realizará con la investigación. Pueden estar orientadas a un subtema, el escenario, método o algún aspecto particular del estudio. Aunque su frecuencia fue menor a la de los objetivos generales, siendo únicamente 21, es un paso prácticamente de tipo obligatorio.

Observar el escenario en donde ocurren con mayor frecuencia el contacto con el inglés en niños migrantes indígenas de Oaxaca.

Determinar cuál es la causa base de la adquisición de préstamos en los niños migrantes indígenas.

Investigar la competencia lingüística entre el español, inglés y su lengua étnica de los niños migrantes oaxaqueños (21).

I) Enunciación de las preguntas de investigación: las preguntas de investigación tienen el papel de plantear las dudas, inquietudes y planteamientos que se desean resolver en la investigación. Tienen una relación estrecha con los objetivos en cuanto a contenido, por lo general se anuncia su llegada después del planteamiento de los objetivos y se enuncia la relación de ellas con los objetivos en algunos casos. Son de tipo obligatorio, pues aparecieron con 26 frecuencias, siendo que solo un trabajo no hizo uso de ellas: 
Por último, y en consideración con los objetivos ya mencionados, se reflexionarán los resultados para llegar a una resolución para responder las incógnitas que a continuación se plantean:

¿Qué consideraciones se deben tomar en cuenta al momento de elegir uno de los distintos significados de un adjetivo y en qué momentos se podría utilizar más de un significado del mismo? ¿Cómo se puede determinar la relevancia de un adjetivo y su importancia dentro del contexto original si su significado en el idioma meta resulta redundante o imposible de traducir directamente? ¿Qué factores externos relacionados con la novela afectan el trabajo del traductor literario? (2).

En algunos casos, las preguntas anteceden a los objetivos y anuncian su relación con las preguntas de investigación:

Así, las preguntas de investigación que guiarán este trabajo son:

1. ¿Cuáles son las características de los textos científicos?

2. ¿ ¿De qué manera impacta la buena o mala redacción de los artículos científicos provenientes de miembros de CICESE?

3. ¿Qué papel emplean los traductores dentro de la publicación de artículos científicos publicados?

Para responder a nuestras preguntas de investigación presentadas arriba, los objetivos del proyecto son. (6).

\section{Discusión}

Los movimientos retóricos del planteamiento del problema que forman parte de los protocolos de tesis de estudiantes de la Licenciatura en Traducción presentan diversidad en las posiciones del texto; fijos o constantes sin ubicación determinada. Los veinte movimientos retóricos son representaciones opcionales que atiende el estudiante como parte de la redacción del 
planteamiento del problema, sin embargo, es necesario puntualizar que los protocolos requieren de elementos específicos como objetivos y preguntas de investigación que definen y orientan al protocolo.

Los movimientos y pasos brindan una estructura específica para mayor claridad con la investigación. Así, el estudiante una vez que identifica la estructura y sus variables redactará con su propio estilo. Es importante concientizar al estudiante de formular la problemática a partir de elementos que permitan evaluarlo; contextos, características e implicaciones dentro del marco ético y objetivo. Si bien, la redacción de los movimientos retóricos parte de las indicaciones del docente, es esencial puntualizar elementos estructurales que deberá contener y de comprender las implicaciones de la argumentación retórica.

Lo investigado enmarca que el protocolo de tesis que se visualiza lograr en la asignatura de Seminario de Investigación ofertada en la etapa terminal de la formación del traductor enuncia los siguientes elementos: 1. Título. 2. Planteamiento del problema dividido en fase histórica y fase personal, en esta última subsección se acomodan los objetivos y las preguntas de investigación. 3. Justificación. 4. Antecedentes. 5. Marco teórico. 6. Metodología dividida en tres secciones: método, técnicas y corpus. 7. Tipo de investigación. 8. Paradigma. 9. Alcance de la investigación. 10. Índice capitular. 11. Cronograma. 12. Bibliografía. 13. Corpus.

Así, atendiendo a la definición de género expuesta en nuestro marco teórico, tenemos que el protocolo de tesis representa muy bien la idea que expresa Martin (1992), quien define que uno de los aspectos que caracterizan a los géneros es su estructura organizada que sigue objetivos. En ese tenor, nos encontramos con un tipo de género que de antemano enuncia su estructura con un énfasis de organización relevante, esperando que el estudiante logre desarrollar cada uno de los elementos enunciados en el anterior párrafo, y además, cada uno de tales elementos persigue un objetivo que no es otro que el estudiante pueda usar cada paso para la construcción de otro género que sería la tesis, siempre y cuando opten por elegir esta modalidad de titulación.

Creemos, que la organización del protocolo de tesis es relevante dentro del marco formativo ya que incide significativamente para proveer la creación de un trabajo más amplio, la tesis. Es claro 
que sin los elementos del protocolo de tesis y, puntualizando en el planteamiento del problema, el estudiante carece y se ausenta de la significación del proceso y conocimiento que deberá tener en el desarrollo del producto esperado. De igual manera es necesario puntualizar que dentro del marco formativo profesional la tesis no representa una obligatoriedad, por ello, si decide no realizar la tesis como parte de las opciones que enmarcan los lineamientos institucionales para la titulación existe un eje relevante. La pertinencia de la formación en la investigación como parte de un marco disciplinar provee al estudiante de una experiencia en la investigación y el género del protocolo aporta competencias y habilidades que serán relevantes para su práctica profesional o formaciones complementarias que el individuo desee realizar a nivel posgrado.

Retomando las definiciones conceptuales sobre movimientos retóricos y pasos retóricos, la fase histórica y la fase personal constituyen etapas funcionales que le dan estructura a todo el planteamiento del problema. Cada movimiento persigue objetivos amplios con diversas segmentaciones textuales, dando lugar a los pasos, éstos a su vez al ser elementos formadores de cada movimiento actúan con mucha libertad en el desarrollo del macromovimiento, que no es otro que el planteamiento del problema.

En el análisis, para el movimiento fase histórica existen pasos que pueden aparecer al principio o tardíamente como la nombrada definición del tema, con la característica que es obligatorio dentro del movimiento en cuestión, pues aparece en todos los protocolos. Cabe mencionar que el movimiento enlista los siguientes pasos obligatorios: Definición del tema, Deber del tema hacia una realización.

Este movimiento a diferencia del movimiento fase personal obtuvo mayor presencia de tipos de pasos, siendo 11 que se reportan. En este punto, es necesario argumentar que el estudiante en el planteamiento del problema describe los elementos específicos de la problemática, así como conceptos donde se presenta la situación actual y la relevancia de la problemática.

El segundo movimiento, fase personal, contiene 9 pasos fundamentales que lo justifican. Siendo que el paso Enunciación del objetivo general es el de mayo obligatoriedad, pues apareció con 27 frecuencias en todos los trabajos. Ante la descripción del objetivo general, se busca que se 
describa de manera clara el resultado que se pretende lograr a través de atender a interrogantes que exponen en el texto.

Le sigue la Enunciación de las preguntas de investigación con 26 frecuencias, enunciación de los objetivos particulares con 21 y propuesta enunciativa con 22 . Los casos que atienden a la pregunta de investigación cumplen con los criterios establecidos donde se exhiben cuestionamientos concretos que evitan la ambigüedad del tema a investigar. Así mismo, los objetivos particulares atienden a una secuencia lógica y jerárquica alineada a la comprensión de las metas establecidas dentro de la investigación.

Podemos sintetizar que un movimiento que se nombra como fase histórica espera como prioridad la definición del tema; en tanto, el movimiento fase personal espera como indispensable para su realización la redacción del objetivo general del trabajo. De ahí presentar como elemento ilustrativo, ante la comprensión de los movimientos, la necesidad de dimensionar los elementos del problema, datos, situaciones y conceptos que deberán estar asociados con la intención de la investigación. De tal manera que se presenten fundamentos que muestren fines de aplicación concretos los cuales se centran en la delimitación especifica de la investigación.

Entre algunas comparaciones que resultan de los dos movimientos, ambos enunciaron: explicación del tema a partir de la revisión de la literatura en la fase histórica, referencia a otros trabajos en la fase personal, como pasos que vinculan el tema con otros antecedentes. El paso descripción de un escenario presenta la misma dinámica que es la descripción del espacio que se investigará. Consideramos que los autores eligen hacia uno y otro movimiento la presencia de estos pasos, al ser opcionales pueden funcionar para uno y otro movimiento, ya que los propósitos comunicativos de cada paso se acomodan a los planteados por los pasos a seguir.

\section{Conclusiones}

A partir de la redacción de pasos y movimientos retóricos, segmentos textuales en el planteamiento del problema del género protocolo de tesis, redactado por estudiantes de la Licenciatura en Traducción de la unidad académica en cuestión; nos damos cuenta cómo la redacción de los 
estudiantes persigue un estilo estructurado en los trabajos analizados. La influencia del docente en esta directriz se hace notoria debido a que la representación de pasos es diversa, de esta forma presentamos como autocrítica que hizo falta en el presente estudio llevar a cabo una entrevista con los docentes para indagar por su formación y cómo esta influye en el estilo de los alumnos.

Los diferentes protocolos analizados al exponer diversidad de pasos indican la buena comprensión del tema y un estilo particular para la redacción de los textos. Hace falta comparar y analizar el resto de las secciones del protocolo como son la justificación, los antecedentes, el marco teórico, la metodología, el tipo de investigación, el paradigma, el alcance de la investigación, cronograma e índice capitular, a fin de obtener otros movimientos retóricos y definir los pasos y movimientos que caracterizan al género en cuestión en el ámbito disciplinar de traductores de la Facultad de Idiomas, UABC. Por otro lado, la carrera de Enseñanza de Lenguas de la misma unidad académica también oferta la materia: Investigación Educativa, que también incide en la propuesta de proyectos de investigación, por lo que se podría en otro proyecto comparar los movimientos y pasos de los protocolos de tesis desarrollados por los estudiantes de ambas carreras.

Finalmente, en cuanto al género académico protocolo de tesis, es un tipo de texto sobre el que existen fuertes carencias en su abordaje como objeto de investigación en el campo de los movimientos retóricos. Si bien, las condiciones actuales no impulsan el desarrollo de la titulación por tesis en la mayoría de las universidades nacionales, debido a que se presentan otras modalidades que desmotivan la realización de una tesis; el protocolo sigue siendo una obligación cuando las carreras imparten las asignaturas que exigen realizar un proyecto de investigación, el cual seguirá siendo fuente para el análisis de pasos y movimientos retóricos de trabajos orientados a una propuesta investigativa.

\section{Referencias}

Ackland, G.M. (2009). A discourse analysis of English and French research article abstracts in Linguistics and Economics. San Diego: Montezuma Publishing Press. 
Biber, D.; Connor, U. y Upton, T. (2007). Discourse on the Move: Using Corpus Analysis to Describe Structure. Amsterdam: John Benjamins.

Castro, C. (2010). Discurso especializado e identidad: el papel de la escritura en el proceso de configuración de la identidad disciplinar en la universidad. En Memorias del XI Encuentro Nacional de Estudios en Lenguas (p.507-519), Tlaxcala: Universidad Autónoma de Tlaxcala.

Castro, C.; Hernández, L.; y Sánchez, M. (2010). El ensayo como género académico: una aproximación a las prácticas de escritura en la universidad pública mexicana. En Parodi (Ed.), Alfabetización académica y profesional en el siglo XXI: Leer y escribir desde las disciplinas (p.49-70). Santiago: Ariel.

Centro de Recursos para la Escritura Académica (2019). Planear y construir borradores. Obtenida el 02 de febrero de 2019 de http://sitios.ruv.itesm.mx/portales/crea/planear/como/planteamiento tesis.htm Ciapuscio, G. (2005). La noción de género en la Lingüística Sistémico Funcional y en la Lingüística Textual. Signos, vol. 38(57), 31-48.

Eggins, S. y Martín, J. (2000). Géneros y registros del discurso. En T. Van Dijk (Ed.), El discurso como estructura y proceso (pp.131-139). Barcelona: Gedisa.

Halliday, M.A.K. (1975). Language as social semiotics: Towards a general sociolinguistics theory. En A. Makkai y V. Becker (Eds.), The first LACUS forum (pp.17-46). Los Angeles, California: Horbeam Press.

Hernández, L. (2011). La pedagogía basada en género de la lingüística sistémico funcional: aprender la lengua, aprender con la lengua y aprender sobre la lengua. Lingüística Mexicana, 1, 61-89.

Hernández, R.; Fernández, C.; y Baptista, C. (2014). Metodología de la investigación. Ciudad de México: McGraw-Hill.

Martin, J.R. (1992). English text: Systems and structure. Filadelfia; Amsterdam: John Benjamins.

Melpopharma (2017). Protocolo de investigación. Obtenida el 02 de febrero de 2019 de http://www.melpopharma.com/investigacion/protocolo-de-investigacion/

Moyano, E. (2007) Enseñanza de habilidades discursivas en español en contexto pre-universitario: Una aproximación desde LSF. Signos, vol. 40 (65), 577-608.

Muñoz, C. (1998). Cómo elaborar y asesorar una investigación de tesis. Ciudad de México: Prentice Hall.

Nwogu, K. N. (1997). The medical research paper: Structure and functions. English for Specific Purposes, vol. $16(2), 119-138$. 
Parodi, G. (2010). La organización retórica del género manual a través de cuatro disciplinas: ¿Cómo se comunica y difunde la ciencia en diferentes contextos universitarios? Boletín de Lingüística, vol. 22 (33), 43-69.

Perales, M.; Sima, E.; Valdez, S. (2014). Movimientos retóricos en las conclusiones de tesis de licenciatura en antropología social: un estudio sistémico-funcional. Escritos Revista del Centro de Ciencias del Lenguaje, vol. 45 (enero-junio 2012), 33-60.

Swales, J.M. (1990). Genre analysis: English in academic and research settings. Cambridge: Cambridge University Press.

Van Dijk, T. (1978). La ciencia del texto. Barcelona: Paidos.

Universidad Veracruzana (S/F). Protocolo de investigación del trabajo recepcional. Obtenida el 02 de febrero de 2019 de https://www.uv.mx/fca/files/2012/09/Protocolo-de-Investigacion.pdf. 